June 15, 2018

IASSNS-HEP-98/2

HUB-EP 98/1

hep-th/9801017

\title{
Brane Dynamics and Chiral non-Chiral Transitions
}

\author{
Ilka Brunner ${ }^{\dagger}$, Amihay Hanany*, Andreas Karch ${ }^{\dagger}$ \\ and Dieter Lüst ${ }^{\dagger}$ \\ ${ }^{*}$ School of Natural Sciences, Institute for Advanced Study \\ Olden Lane, Princeton, NJ 08540, USA \\ ${ }^{\dagger}$ Institut für Physik, Humboldt Universität Berlin \\ Invalidenstr. 110 \\ 10115 Berlin, Germany
}

\begin{abstract}
We study brane realizations of chiral matter in $N=1$ supersymmetric gauge theories in four dimensions. A "cross" configuration which leads to "flavor doubling" is found to have a superpotential. The main example is realized using a special "fork" configuration. Many of the results are found by studying a $S U \times S U$ product gauge group first. The chiral theory is then an orientifold projection of the product gauge group. An interesting observation in the brane picture is that there are transitions between chiral and non chiral models. These transitions are closely related to small instanton transitions in six dimensions.
\end{abstract}




\section{Introduction}

The past few years have seen tremendous progress in our understanding of non-perturbative effects in supersymmetric field theory and string theory. Many insights into gauge theories have emerged by using D brane techniques. A particular brane configuration where D branes are suspended between NS branes was first used in [1] and later used to study theories with eight or four supercharges in various dimensions. Here, the gauge theory under consideration is realized as the world volume theory of the lowest dimensional D brane in the setup. Fundamental matter can be included by adding higher dimensional D branes. This basic setup can be modified by including further ingredients such as orientifolds. Of particular interest are configurations corresponding to $N=1$ supersymmetric theories in four dimensions. Here, one success was that Seiberg's duality [2], could be reproduced from the brane picture [3]. However, a brane configuration giving $N=1$ differs from a setup leading to $N=2$ only by a rotation angle of the NS branes with respect to each other. Therefore, a brane configuration leading to $N=1$ locally looks like an $N=2$ configuration. An important difference between $N=1$ and $N=2$ is chiral symmetry. A priori we only see a diagonal subgroup of the full chiral symmetry group in the brane

picture. In [4] it was suggested that the full chiral symmetry is restored if the flavour giving D branes are broken in two pieces by an NS brane whose world volume directions coincide with world volume directions of the D6 branes. More generally, chiral multiplets correspond to "half-branes" ending on NS branes. Anomaly cancellation in field theory translates to RR charge conservation in the string theory context [5]. In this paper we will study the localization of chiral matter in $N=1, d=4$ in some more detail. In the first section we look at situations where D6 branes touch D4 branes at points where also NS' branes are localized. Here, we observe the presence of additional multiplets from the breaking of the D6 and D4 branes, as well as a nonzero superpotential. In the next section we use this observation in the context of product gauge groups. After that we specialize to configurations which are symmetric with respect to the location of an NS brane. Here it is possible to introduce orientifolds in various ways. One of our models is a chiral theory with chiral multiplets in the fundamental, symmetric and antisymmetric representation. This model has also been studied recently in a paper by Landsteiner, Lopez and Lowe [6], which appeared while this work was in progress. Other realizations of chiral gauge theories from branes can be obtained by looking at brane configurations in an orbifold background $[7,8]$. Finally, we show in the brane picture that there exists a phase transition from this chiral model to a non-chiral model. This is a very striking effect, in which the continuation past infinite coupling of a non-chiral theory turns out to be the chiral model. Such a transition was studied recently in a T-dual model in the 
brane realization of six dimensional theories [9]. From a T-dual point of view, of the world volume theory on the D6 branes this corresponds to a small instanton transition. Thus, the two field theory transitions are a consequence of the same brane motion, which puts them on the same universality class. Such chirality changing trasitions have been previously considered from geometric considerations in $[10,11]$.

Let us set some notation and recall simple facts which we will use throughout this paper. We work in Type IIA superstring theory. The various branes considered in this paper will be NS brane which spans directions 012345, D4 brane along the directions 01236, D6 brane with $0123789, \mathrm{NS}^{\prime}$ with $012389, \mathrm{D} 6^{\prime}$ with 0123457 . The presence of these branes breaks supersymmetry to 4 supercharges and lead to a four dimensional $N=1$ supersymmetic gauge theory on the world volume of the $\mathrm{D} 4$ branes. These branes are finite in the $x^{6}$ direction and reduction on this interval leads to the four dimensional theory sitting in 0123. $S O(1,9)$ space time Lorentz symmetry is broken to $S O(1,3) \times S O(2) \times S O(2)$. The first factor is the Lorentz symmetry of the four dimensional theory. The two other $S O(2)$ factors act on 45 and 89 directions and are R-symmetries of the four dimensional theory. A rotation of the NS branes and the D6 branes along the 45-89 directions does not break further the supersymmetry and we will use this property to add more parameters to the theory studied.

\section{Localization of Chiral Matter in Space}

In this section we will study a particular configuration in which chiral matter with a nontrivial superpotential is localized in spacetime. This configuration serves as a building block for the models that are studied in this paper and for other generalizations. 


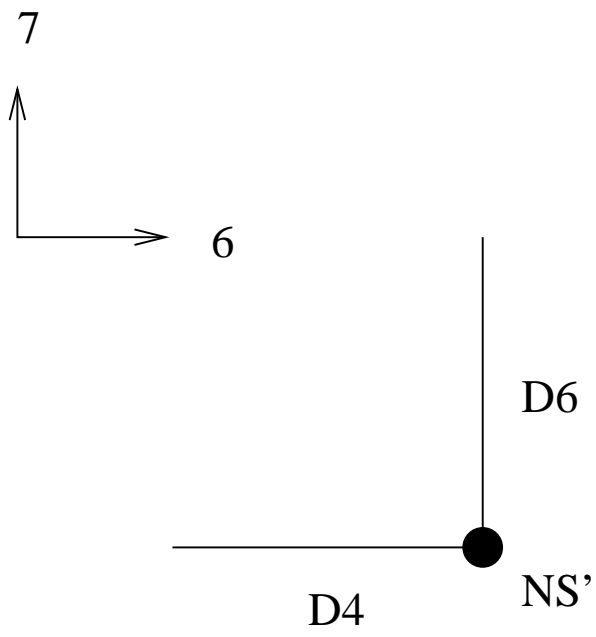

Figure 1: A configuration of branes in which a single chiral field is localized in space time. A D6 brane ending on a $\mathrm{NS}^{\prime}$ brane meets a D4 brane which also ends on the $\mathrm{NS}^{\prime}$ brane.

Let us start by recalling some of the relevant configurations already studied. In [4], it was suggested that chiral symmetry is enhanced at special points were a D6 brane meets a NS' brane. This led to a localization of chiral matter in the configuration of figure 1 which arises at the intersection of a D4 brane and a D6 brane which end on NS' brane [5]. Evidence for this proposal using various arguments were given in $[12,5,13]$. 


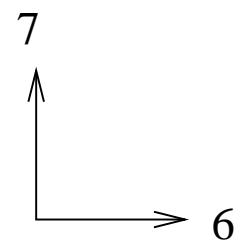

D6

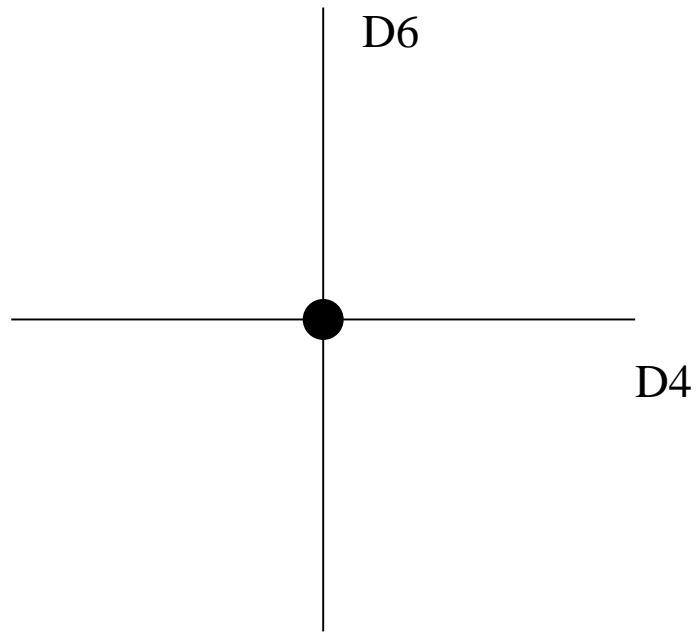

Figure 2: A "cross" configuration. An intersection of a D4 brane, a D6 brane and a $\mathrm{NS}^{\prime}$ brane. There are four chiral fields, two chiral bi-fundamentals and a superpotential.

We wish to use this identification to study the configuration given in figure 2 . First let us recall that when a D6 brane meets a D4 brane there is a massless hypermultiplet which transforms under the $U(1) \times U(1)$ gauge groups which sit on the $\mathrm{D} 4$ and $\mathrm{D} 6$ branes. The number of supersymmetries for such a configuration is 8 and so there is a superpotentail which is restricted by the supersymmetry to be $(m-x) \tilde{Q} Q$, where $m$ is the 45 position of the D6 brane, $x$ is the 45 position of the $\mathrm{D} 4$ brane and $\tilde{q}, q$ transform as $(-1,1)$ and $(1,-1)$, respectively.

We can slowly tune the position of a $\mathrm{NS}^{\prime}$ brane to touch the intersection of the $\mathrm{D} 4$ and $\mathrm{D} 6$ branes. The number of supersymmetries locally is now 4 . At this point, both the D4 and the D6 branes can break and the gauge symmetry is enhanced to $U(1)_{u} \times U(1)_{r} \times U(1)_{d} \times$ $U(1)_{l}$. The $u, d$ indices correspond to the two parts of the $\mathrm{D} 6$ branes and the $l, r$ indices correspond to the two parts of the D4 branes. As usual for the transitions involving NS branes which lead to breaking of the D branes, we should look for an interpretation as a Higgs mechanism. Since, the number of vector multiplets is increased by two, we need to look for two more massless chiral fields. By applying the configuration of figure 1, we see that we have four copies of chiral multiplets $\tilde{Q}, Q, \tilde{R}, R$. They carry charges $(1,-$ 
$1,0,0),(0,1,-1,0),(0,0,1,-1),(-1,0,0,1)$, respectively under the gauge groups. In addition there are bi-fundamental fields for the intersection of the two new D4 branes, $\tilde{F}, F$ with charges $(0,1,0,-1)$ and $(0,-1,0,1)$, respectively. Two more bi-fundamental fields come from the two new D6 branes. For the moment, we will ignore the bi-fundamentals for the D6 branes, since they have six dimensional kinetic terms and so are not dynamical for the four dimensional system. All together we have $\left(\begin{array}{l}4 \\ 2\end{array}\right)$ possible fields coming from the intersection of each pair of branes from possible 4 .

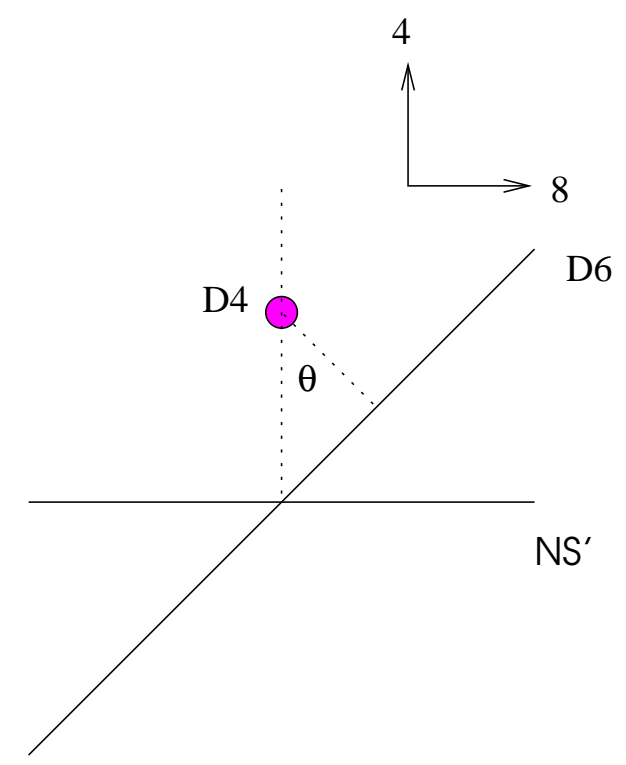

Figure 3: A deformation of the "cross" configuration by reconnecting the two D4 branes and moving the D4 brane in the 45 direction. The dotted lines do not represent branes.

The system has 4 supercharges and we can not exclude the possibility of a superpotential. We can write down a superpotential of the form

$$
a \tilde{Q} \tilde{F} R+b \tilde{R} F Q .
$$

The coefficients $a$ and $b$ are determined by looking at various deformations of this configuration. One possible deformation is a rotation of the D6 branes in the 45-89 direction. This rotation does not break further the supersymmetry. There is one angle between the D6 branes and the $\mathrm{NS}^{\prime}$ brane. One can think that there may be two angles, one for the upper D6 brane and one for the lower D6 brane. However, RR charge conservation of the D6 branes implies that they must be parallel to each other and so the angles are the same. Let us choose an orientation by fixing the $\mathrm{NS}^{\prime}$ brane and let $\theta$ be the angle between 
the rotated $\mathrm{D} 6$ brane and the $\mathrm{NS}^{\prime}$ brane. It is defined in such a way that for $\theta=0$ we recover the $\mathrm{D} 6$ brane whereas for $\theta=\frac{\pi}{2}$ we get the $\mathrm{D} 6^{\prime}$ brane.

Let us reconnect the two D4 branes and move them in the 45 directions as in figure 3 . This motion corresponds to an expectation value for the bi-fundamental fields $\tilde{F}$ and $F$. From equation (1) we see that this gives a mass to all four fields $\tilde{Q}, R, \tilde{R}, Q$. The mass is given by the distance between the two $\mathrm{D}$ branes, $F \cos \theta$. The symmetry of the two objects implies that $a$ and $b$ are of the same magnitude up to a sign. This is determined to be a minus sign by a motion of the D6 branes along the $x^{6}$ direction. The final superpotential is

$$
\cos \theta \tilde{Q} \tilde{F} R-\cos \theta \tilde{R} F Q .
$$

In this discussion we have omitted possible chiral fields associated to the motion of the D4 branes along the $\mathrm{NS}^{\prime}$ branes. Inclusion of these fields gives rise to interactions which are restricted by supersymmetry with 8 supercharges and contribute appropriate terms to the superpotential.

We can now generalize the construction to configurations with more than one brane of a given type. Suppose we have $n_{l}\left(n_{r}\right)$ D4 branes to the left (right) of the NS' brane and $n_{u}\left(n_{d}\right)$ D6 branes above (below) the $\mathrm{NS}^{\prime}$ brane. First, $n_{u}=n_{d}=n$ by RR charge conservation. The gauge and global symmetries are $U(n)_{u} \times U\left(n_{r}\right) \times U(n)_{d} \times U\left(n_{l}\right)$, under

which the fields, $\tilde{Q}, Q, \tilde{R}, R$ transform as $(\square, \bar{\square}, 1,1),(1, \square, \bar{\square}, 1),(1,1, \square, \bar{\square}),(\bar{\square}, 1,1, \square)$, together with bi-fundamental fields $\tilde{F}, F$ which transform under $(1, \square, 1, \bar{\square})$ and $(1, \bar{\square}, 1, \square)$, respectively. The superpotential is given by equation (2). We can break the global symmetries $U(n) \times U(n)$ by rotating each $\mathrm{D} 6$ brane independently. This breaks the global symmetry generically to $U(1)^{2 n}$. The different angles correspond to the eigenvalues of the Yukawa coupling matrix in equation (2).

Equipped with this configuration and its superpotential, let us revisit the brane configuration for products of $S U$ gauge groups.

\section{Product Gauge Groups}

In this section we will use the "cross" configuration studied in the previous section to study a system which describes product of gauge groups. This will help us understand the doubling of fields at the intersection between the two gauge groups which was studied in section 2.9 of [12]. 


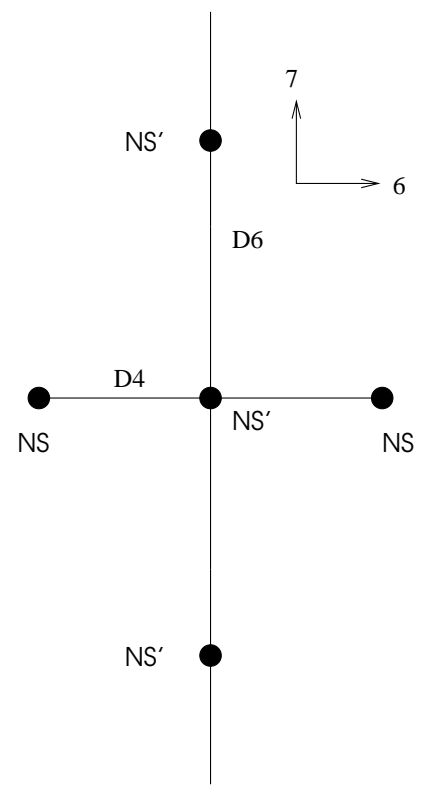

Figure 4: A demonstration of the "doubling" of matter for a brane configuration of a product of two gauge groups. The upper and lower NS' branes are put sufficiently far from the $4 \mathrm{~d}$ system. They are introduced to study the six dimensional system which governs the global symmetry of the four dimensional thoery.

\subsection{Flavor Doubling}

Consider, as in figure 4 , a system of NS-NS'-NS branes positioned in the $x^{6}$ direction in this order. Let $N_{c}$ D4 branes be stretched between the left NS brane and the NS' brane, and $N_{c}^{\prime} \mathrm{D} 4$ branes be stretched in between the right NS brane and the $\mathrm{NS}^{\prime}$ brane. Let $N_{f}$ D6 branes be positioned at the $x^{6}$ position of the $\mathrm{NS}^{\prime}$ brane, in such a way that they touch the D4 branes and give rise to massless multiplets. This is precisely the "cross" configuration, figure 2 , studied in the previous section. Using the results from this section, the gauge theory and matter are $S U\left(N_{c}\right) \times S U\left(N_{c}^{\prime}\right)$ with matter $4 N_{f}$ chiral fields: $N_{f}$ flavors $\tilde{Q}, Q$ in $\left(1, N_{c}^{\prime}\right)$, and $N_{f}$ flavors $\tilde{R}, R$ in $\left(N_{c}, 1\right)$. There are two bi-fundamental chiral fields $\tilde{F}, F$ in the $\left(N_{c}, \overline{N_{c}^{\prime}}\right)$ and its complex conjugate, $\left(\overline{N_{c}}, N_{c}^{\prime}\right)$. There is a superpotential of the form

$$
\tilde{Q} \tilde{F} R-\tilde{R} F Q .
$$

As usual, the 45 positions of the D6 branes give rise to masses for both types of flavors. To do that, the global symmetry is broken explicitely to $S U\left(N_{f}\right)$. The brane interpretation is clear. We reconnect the upper and lower D6 branes to form a single D6 brane which 
moves in the 45 direction and gives masses which transform in the adjoint representation of the new $S U\left(N_{f}\right)$ group. A more confusing issue is with the $x^{6}$ motion of the D6 branes. It is easy to see that such a motion to the left (right) gives mass to the $R(Q)$ fields and leaves the $Q(R)$ fields massless. How can this be included in the superpotential?

To solve this puzzle, it is convenient to recall the six dimensional system which controls the global symmetries of our system $[14,15,5,9]$. This is a six dimensional $S U\left(N_{f}\right) \times S U\left(N_{f}\right)$ with a matter $f$ in the bi-fundamental representation. It is convenient to assume the existence of two $\mathrm{NS}^{\prime}$ brane far along the $x^{7}$ direction in both sides of the $\mathrm{NS}^{\prime}$ at the origin of $x^{7}$, as in figure 4 .

Then, anomaly cancellation in six dimensions requires additional $N_{f}$ fundamental fields for each $S U\left(N_{f}\right)$ group, as can be read off from the brane configuration.

The main point in introducing the six dimensional theory is that its moduli space is mapped to the space of parameters of the four dimensional theory. Let us look at a particular configuration, in which an infinite D6 brane reconnects and moves in the 456 direction. This corresponds to a flat direction for the bi-fundamental field $f .{ }^{1}$ There are four scalars associated with such a motion. These are the 456 distance to the $\mathrm{NS}^{\prime}$ brane and the reduction of the $A_{7}$ component of the gauge field which lives on the worldvolume of the D6 brane. They decompose into 45 chiral field, $m$, and $\tilde{m}=x^{6}+A_{7}$ chiral field. As said above, the 45 expectation values are mapped to bare masses of both the $Q$ fields and the $R$ fields. The puzzle raised in the last paragraph is now solved by introducing the new mass term coming from the six dimensional $\tilde{m}$ chiral field. D6 branes with positive $x^{6}$ position contribute to the superpotential $\tilde{m} \tilde{R} R$. D6 branes with negative $x^{6}$ position contribute $\tilde{m} \tilde{Q} Q$ to the superpotential. We see that there are two patches in which the superpotential looks different. The transition between the two regions is smooth since the difference in the contribution to the two superpotentials vanishes there.

We can generalize further the system by introducing $k$ left NS branes and $k^{\prime}$ right NS branes. As suggested in [3], this includes the addition of two adjoint fields, $x$ and $y$, for the left and right group, respectively. The superpotential then becomes

$$
x^{k+1}+y^{k^{\prime}+1}+(x-y) \tilde{F} F+\tilde{R}(m-x) R+\tilde{Q}(m-y) Q+\tilde{Q} \tilde{F} R-\tilde{R} F Q+W_{p},
$$

where $W_{p}$ is defined in two different regions,

$$
\begin{aligned}
W_{p} & =\tilde{m} \tilde{Q} Q, & & x^{6}<0 \\
& =\tilde{m} \tilde{R} R, & & x^{6}>0
\end{aligned}
$$

\footnotetext{
${ }^{1}$ The gauge invariant operator is actually a meson which includes the lower and upper flavors of the six dimensional theory, with the bi-fundamental fields.
} 
We use this superpotential in the next sections to determine more complicated setups.

\subsection{Duality for the Product Gauge Group}

We proceed by recalling some of the properties in the models which were studied in [4]. The brane configuration is slightly more general than the one introduced in the previous section. However, there is also a simplification. For the moment, we avoid the issue of "flavor doubling" and keep the D6 branes away from the $\mathrm{NS}^{\prime}$ brane in the $x^{6}$ position. The D6 branes give rise to flavors of one of the gauge groups, not both, depending on their $x^{6}$ position.

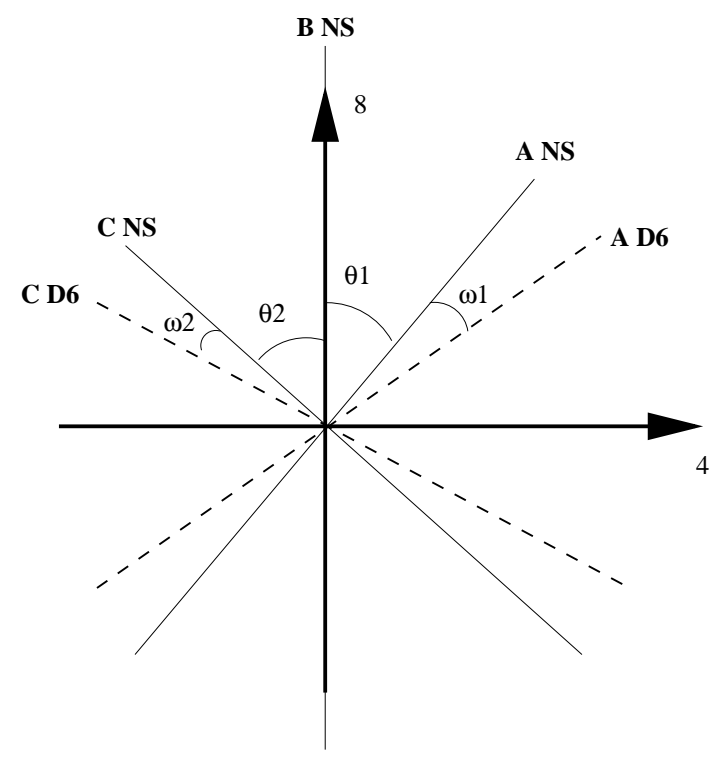

Figure 5: Notation for the angles in the product group setup.

First, we allow for a general number of NS, NS' and NS branes. For convenience, we will denote them A, B and C, respectively. Second, we will allow for general angles in the 45-89 space. The names NS brane and NS' brane are no longer useful. Instead we use the general name NS branes, keeping in mind that each NS brane has a general angle which specifies the orientation in 45-89 space. The configuration is depicted in figure 5 . In this figure, we also draw the angles for the various branes. The $\mathrm{B}$ branes remain $\mathrm{NS}^{\prime}$ branes by a choice of the origin for the angular variable. Let $\left(k, k^{\prime}, k^{\prime \prime}\right)$ denote the number of $\mathrm{A}, \mathrm{B}$ and $\mathrm{C}$ type NS branes respectively. According to [4] the $(k, k, k)$ case leads to a 
superpotential

$$
W=m_{1} X_{1}^{k+1}+m_{2} X_{2}^{k+1}+X_{1} \tilde{F} F+X_{2} \tilde{F} F+\lambda_{1} Q X_{1} \tilde{Q}+\lambda_{2} Q^{\prime} X_{2} \tilde{Q}^{\prime}
$$

while the $(k, 1, k)$ case leads to

$$
W=-\frac{1}{2}\left(\frac{1}{m_{1}}+\frac{1}{m_{2}}\right)(F \tilde{F})^{k+1}+\lambda_{1} Q X_{1} \tilde{Q}+\lambda_{2} Q^{\prime} X_{2} \tilde{Q}^{\prime} .
$$

In the following, we identify these superpotentials by their triple number, $(\cdot, \cdot, \cdot)$. The coefficients in the superpotential are determined in terms of the angles as

$$
\begin{aligned}
\lambda_{1} & =\sin \omega_{1} \\
\lambda_{2} & =\sin \omega_{2} \\
m_{1} & =\tan \theta_{1} \\
m_{2} & =\tan \theta_{2} .
\end{aligned}
$$

To discuss the duality in these gauge theories it will be easiest to focus on the case where $\omega_{1}=\omega_{2}=0$, as it was discussed in [4]. Choosing non-zero values for these angles amounts to turning on the $Q X_{1} \tilde{Q}$ perturbation.

The magnetic theory is obtained from this by switching the two end NS branes while keeping the center NS brane in place. The resulting gauge group is determined by charge conservation and can be read of from the brane configurations using the linking numbers of [1].

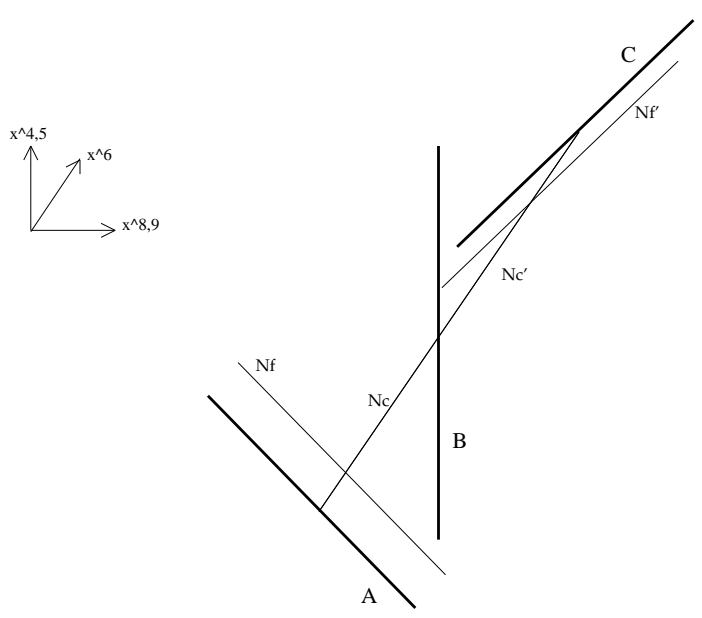

Figure 6: The product gauge group considered in [4]. The thick lines are NS branes at arbitary angle in 45-89 direction. The D6 branes are parallel to the $\mathrm{A}$ and $\mathrm{C}$ NS branes. 


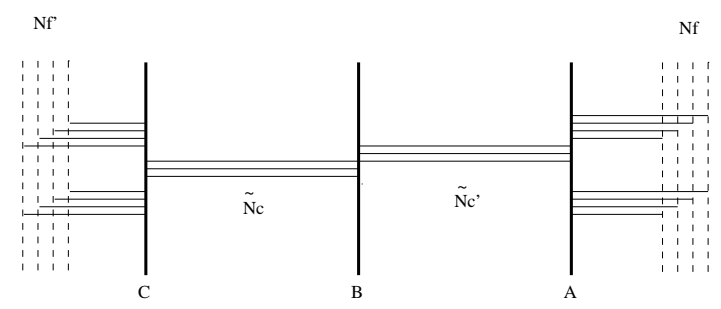

Figure 7: The dual of figure 6 , done by reversing the order of NS and D6 branes [4].

The linking number of a NS brane is given by $L_{5}=\frac{1}{2}\left(n_{6 L}-n_{6 R}\right)+\left(n_{4 R}-n_{4 L}\right)$ and that of a D6 brane by $L_{6}=\frac{1}{2}\left(n_{5 L}-n_{5 R}\right)+\left(n_{4 R}-n_{4 L}\right)$ where $n_{p L, R}$ denotes the number of $p$ branes to the left(right) of the brane under consideration. However parallel branes do not contribute to the linking number [4]. With this, it is easy to find that for $(k, 1, k)$ the dual gauge group is $S U\left(k N_{f}+(k+1) N_{f}^{\prime}-N_{c}^{\prime}\right) \times S U\left(k N_{f}^{\prime}+(k+1) N_{f}-N_{c}\right)$ and for $(\mathrm{k}, \mathrm{k}, \mathrm{k})$ the dual gauge group is $S U\left(k N_{f}+2 k N_{f}^{\prime}-N_{c}^{\prime}\right) \times S U\left(k N_{f}^{\prime}+2 k N_{f}-N_{c}\right)$. For general $\left(k, k^{\prime}, k^{\prime \prime}\right)$ the dual is $S U\left(k^{\prime \prime} N_{f}+\left(k+k^{\prime}\right) N_{f}^{\prime}-N_{c}^{\prime}\right) \times S U\left(k N_{f}^{\prime}+\left(k+k^{\prime \prime}\right) N_{f}-N_{c}\right)$, even though the field theory interpretation of this brane configuration is not clear at this stage.

\section{Chiral Gauge Theories}

We now turn to study a particular chiral gauge theory. All the information collected so far in the previous sections will prove to be useful in studying this system. Let us first give the general setup.

\subsection{The Setup}

Let us consider the same setup as in the previous section. In addition we include an orientifold 6 plane. As with the D6 and NS branes supersymmetry allows for O6 planes with arbitrary orientation in the 4589 direction. Using the by now familar notation we will call an orientifold parallel to the D6' brane (that is stretching along 0123457) an $\mathrm{O}^{\prime}$ and an orientifold parallel to the D6 an O6. To fix the overall orientation we will always take the center (B) brane to be an $\mathrm{NS}^{\prime}$ brane. In order to be able to introduce the orientifold plane, the setup has to be symmetric with respect to the orientifold. This means in particular: 
- $N_{c}=N_{c}^{\prime}$

- $N_{f}=N_{f}^{\prime}$

- $\theta_{1}=-\theta_{2}$ and $\omega_{1}=-\omega_{2}$ (the A branes have to be mirrors of the $\mathrm{C}$ branes)

- the number of $\mathrm{A}$ branes and $\mathrm{C}$ branes has to be equal, that is we only consider $\left(k, k^{\prime}, k\right)$ configurations

- one can only include an $\mathrm{O} 6$ or an $\mathrm{O}^{\prime}$ (the $\mathrm{B} \mathrm{NS}^{\prime}$ brane, carrying one unit of NS charge, has to be self-mirror)

A different way to project to $S O$ and $S p$ groups would be to introduce an O4 plane. This possibility was discussed in [16].

\subsection{Identification of the gauge group}

Consider the following setups involving O6 planes. 

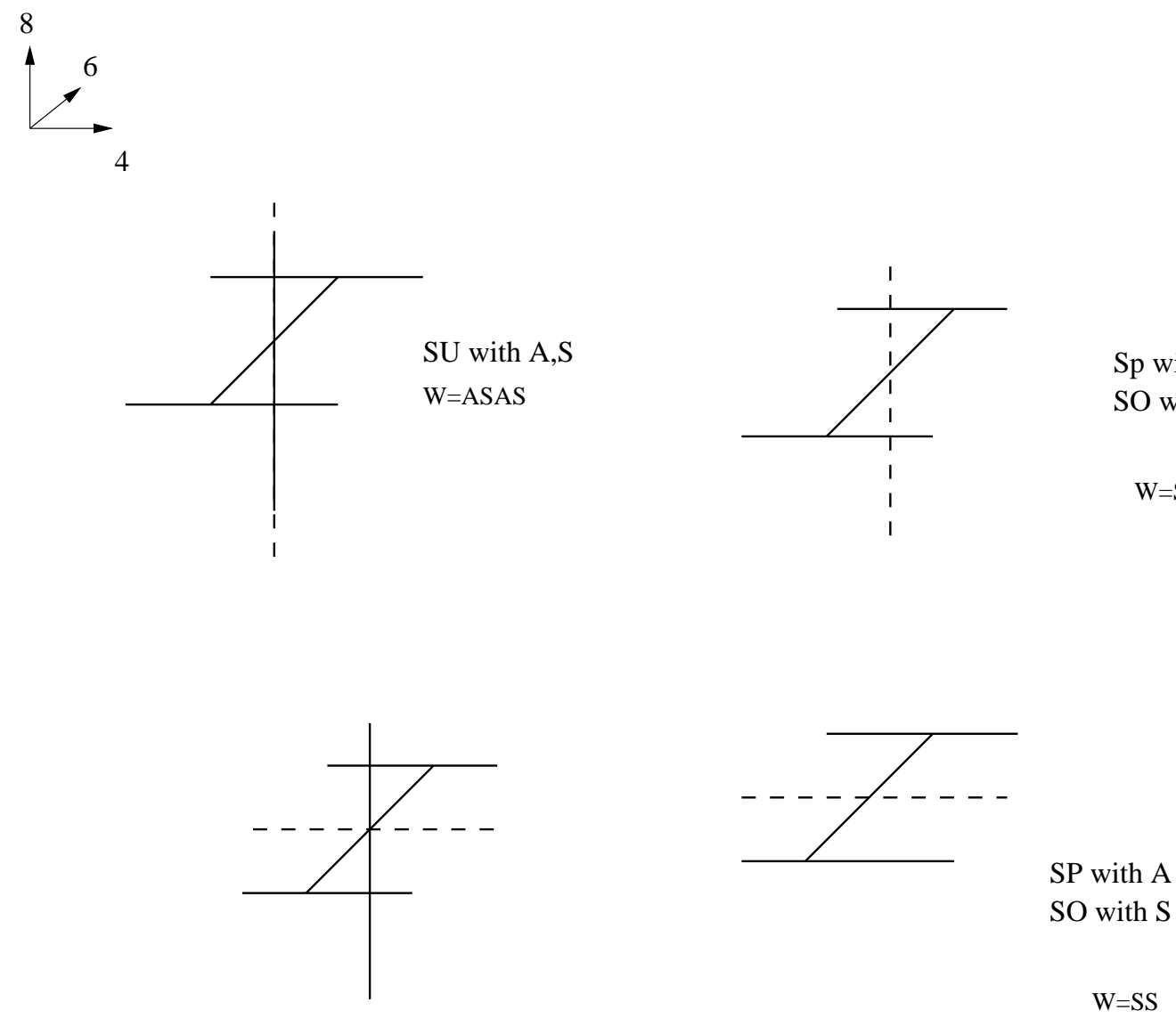

SU with S + conj.
SU with A + conj.

Figure 8: Theories with orientifolds and their deformations

On the left in figure 8 we find the two possible projetions of the product gauge group setup, the right hand side displays two possible setups involving just 2 NS branes and an orientifold, which arise as possible deformations of the theories on the left, as we will see in the following. Let us briefly summarize the resulting gauge groups and matter contents. A more detailed discussion of this identification will be presented in the following sections.

In the two pictures on the left the two $S U$ factors are identified under the orientifold projection. One adjoint field is present, whose mass is given by the angle $\theta$ between NS and $\mathrm{NS}^{\prime}$ brane. In addition there are degrees of freedom that gave rise to bifundamentals in the product gauge groups. In the following two sections we will show that in the orientifolded theories they will give rise to a complex symmetric and an antisymmetric tensor for the $\mathrm{O} 6$ and one flavor of symmetric or antisymmetric tensors for the $\mathrm{O}^{\prime}$, depending on the sign of the projection. 
The configuration in the upper right corner of figure 8 is just the $N=2$ setup analyzed in [17]. The corresponding gauge group is $S O(S p)$ depending on the sign of the orientifold projection. Rotating the NS branes breaks $N=2$ to $N=1$ by giving a mass to the adjoint chiral multiplet in the $N=2$ vector multiplet. We are left with an $S O(S p)$. Note

that this mass is already infinite at $\theta=\frac{\pi}{4}$, since this time it is given by the angle between the outer NS branes which is twice the angle $\theta$ between outer NS and NS ${ }^{\prime}$ that determined the mass before. Rotating further we claim that instead of the adjoint tensor that became infinitely heavy a new tensor with the opposite symmetry properties is coming down from infinite mass. We will find evidence for this from comparing the branches of the various brane configurations with results from field theory. Note that if we were dealing with $\mathrm{D}$ branes instead of the NS branes the rotation we performed would precisely change their worldvolume gauge theory from $S O$ to $S p$. It's therefore reasonable to assume that something similar happens to the NS branes, too. This way we identify the gauge group corresponding to the brane configuration in the lower right corner of figure 8 to be a $S O(S p)$ gauge theory with a symmetric (antisymmetric) tensor. Note that we this way reproduced all the $A_{1}$ theories in the classification of [18].

\section{3 $S U$ gauge groups with 2-index tensors and adjoint}

\subsubsection{The brane configuration}

Let us first discuss the case of an $\mathrm{O}^{\prime}$ plane. We have to distinguish two cases, since the orientifold can carry two different charges, leading to different projections. The orientifold projection leads to the following gauge group and matter content: since the left gauge group is identified with the right gauge group, we are only left with one $S U\left(N_{c}\right)$ gauge group. The two adjoint fields are also identified and we are only left with one adjoint $X=X_{1}=X_{2}$. Some of the degrees of freedom that lead to the bifundamentals are projected out since the corresponding strings stretch across the orientifold, leaving a single flavor of a symmetric or an antisymmetric tensor (that is the tensor and its complex conjugate). The superpotential for an $\mathrm{O}^{\prime}$ for $(k, k, k)$ with a positive $\mathrm{RR}$ charge can easily be obtained from $(7)$ to be

$$
W=m X^{k+1}+\lambda Q X \tilde{Q}+X S \tilde{S}
$$

where $m=m_{1}$ and $\lambda=\lambda_{1}$ can be obtained from the angles as above and $S, \tilde{S}$ denotes the symmetric flavor obtained from the bifundamentals after projection. The same superpotential with $A$ substituted for $S$ is obtained if we use the other projection, with negative $\mathrm{O} 6^{\prime}$ charge, to obtain the antisymmetric tensor. 


\subsubsection{Flat Directions}

We will only discuss the case with the symmetric flavor (corresponding to the $\mathrm{O}^{\prime}$ ' with positive charge), the antisymmetric tensor can be treated in a completely analogous fashion, replacing $S O$ with $S p$ groups. The field theory was analyzed in [19] for the superpotential $(1,1,1)$ and in [18] for the superpotential $(k, k, k)$. For simplicity we will focus just on the $(1,1,1)$ case. We will only consider the configuration with $\lambda=0$. First consider the case with $\theta=\pi / 2$. The adjoint becomes infinitely heavy and hence all quartic superpotentials vanish. In the brane picture the $\mathrm{A}$ and $\mathrm{C}$ branes are parallel. This time, the D4 branes are free to move in the 4,5 direction along the A and C NS branes. In the field theory this new branch opens up since the $(S \tilde{S})^{2}$ term vanishes and now $S$ and $\tilde{S}$ are free to get vevs of the form

$$
\langle S\rangle=\left(\begin{array}{llll}
x_{1} & & & \\
& x_{2} & & \\
& & \ddots & \\
& & & x_{n}
\end{array}\right),
$$

and

$$
\langle\tilde{S}\rangle=\left(\begin{array}{cccc}
\tilde{x_{1}} & & & \\
& \tilde{x_{2}} & & \\
& & \ddots & \\
& & & \tilde{x}_{n}
\end{array}\right)
$$



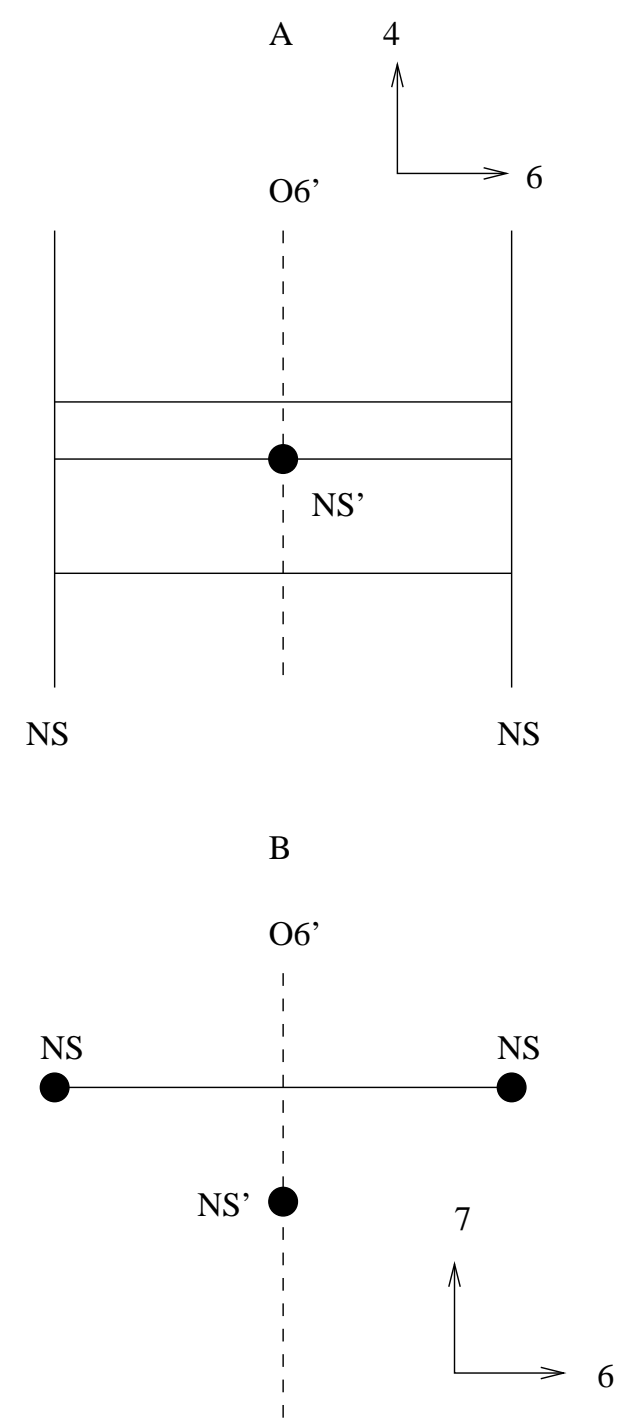

Figure 9: Patterns of flat directions for the $(1,1,1)$ theory with no superpotential. In figure A we see the motion of D4 branes along the NS branes in the 45 directions which leads to either $S U$ or $S O$ theories. In figure $B$ we see a slice in the 7 direction in which the NS branes may be moved away from the $\mathrm{NS}^{\prime}$ branes, leading to a non-zero constant, $r$.

with $\left|x_{i}\right|^{2}-\left|\tilde{x}_{i}\right|^{2}=r, r$ being a constant, generically breaking $S U\left(N_{c}\right)$ completely [19]. The $z_{i}=x_{i} \tilde{x}_{i}$ label the 4,5 position of the $i$-th brane. If $n$ branes have $z_{i}=0$ they coincide at the origin (the $\mathrm{NS}^{\prime}$ position) and yield an unbroken $S U(n)$ gauge group with the original matter content. If $p$ coincide at non-zero $z$, they give an unbroken $S O$ subgroup, which is obvious from both brane and field theory points of view. See figure 9 A. The constant $r$ is identified with the distance between the NS branes and the $\mathrm{NS}^{\prime}$ brane, as can be seen in figure $9 \mathrm{~B}$. 
For general values of $\theta$ the mass term for $X$ and the resulting quartic superpotential, $(\tilde{S} S)^{2}$, do not allow for any Coulomb branch. However there are several Higgs branches. The mesonic branches arise in the standard fashion by splitting the D4 branes between the D6 branes. The gauge group is broken to the same model with a lower rank gauge group.

More interesting are the baryonic branches of the theory. In the brane picture baryonic branches are associated with motion of NS branes in the 7 direction. Consider the flat direction along which the baryon $S^{n} Q^{N_{c}-n} Q^{N_{c}-n}$ gets an expectation value. The field theory analysis was performed in [19]. Along this flat direction the gauge group is broken to $S O(n)$ with a symmetric tensor and a superpotential $W=m X^{2}$. What happened in the brane language is that we moved the center $\mathrm{NS}^{\prime}$ brane along the 7 direction. Thereby we obtain a configuration of parallel NS branes with a parallel orientifold in between, giving rise to an $S O$ gauge group with a symmetric tensor as claimed.

\subsubsection{Non-Abelian Duality}

Similarly, one can carry over the construction of the non-Abelian duality from the discussion of the product gauge group in section 3.2. Consider the $(1,1,1)$ case. As above, the dual gauge group is most easily obtained for the case $\lambda=0$, that is with the D6 branes parallel to the outer NS branes, $\omega=0$. The only difference is that we have to take into account the orientifold plane. Since it is charged like \pm 4 D6 branes we get an additional \pm 4 in the dual gauge group. Since we can not move the O6 to the outside of the NS branes like we did with the D6 branes, the O6 charge appears without the factor of 3 and we get as the dual gauge group an $S U\left(3 N_{f} \pm 4-N_{c}\right)$ gauge group with the same charged matter content and several meson fields in agreement with field theory expectations.

If we move the D6 branes on top of the orientifold and hence also on top of the center NS brane, the issue of flavor doubling as before arises. For a positive charge $\mathrm{O}^{\prime}$, with the additional terms in equation (1), the superpotential takes the form

$$
W=S \tilde{Q} \tilde{Q}+\tilde{S} Q Q
$$

where we now have $2 N_{f} Q$ fields instead of the $N_{f} Q$ fields considered so far. The global symmetry is enhanced from $S U\left(N_{f}\right) \times S U\left(N_{f}\right)$ to $S O\left(2 N_{f}\right) \times S O\left(2 N_{f}\right)$ as expected for $\mathrm{D}$ branes coinciding on top of an orientifold. Similarly, for a negative charge $\mathrm{O}^{\prime}, S$ is replaced by $A$ and the global symmetry is enhanced to $S p\left(N_{f}\right) \times S p\left(N_{f}\right)$. 


\subsection{The Chiral Theory}

\subsubsection{The brane configuration}

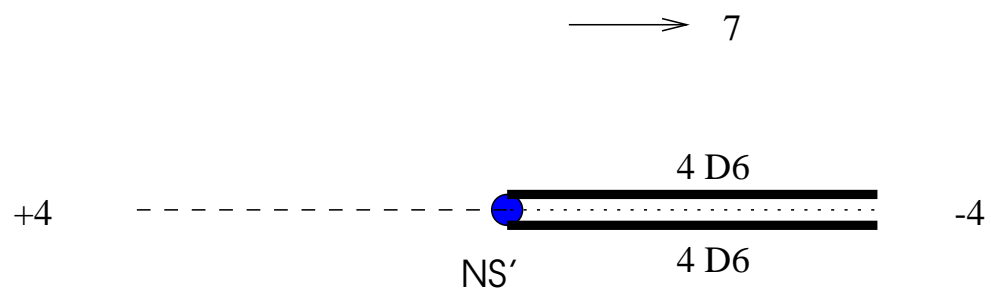

Figure 10: An O6 plane and a $\mathrm{NS}^{\prime}$ brane, the "fork". The sign of the O6 is changed as it crosses the NS' brane. Dashed line represents O6 with charge +4 . Dotted line represents O6 with charge -4 . Anomaly cancellation requires the existence of semi-infinite D6 branes. The 4 D6 branes and their images, are represented by fat solid lines.

The story becomes more complicated once we consider the case of an O6 plane. As considered in [20] an O6 changes sign when passing through an NS' brane. Anomaly freedom of the corresponding $6 \mathrm{~d}$ field theory requires this object to be accompanied with 4 physical half D6 branes and their mirrors stuck on the negatively charged half of the O6. In total we have the following picture, as in figure 10, (reading along the 7 direction): from infinity running inwards we have an $\mathrm{O} 6$ of charge +4 . At $x_{7}=0$ we find the $\mathrm{NS}^{\prime}$ brane with the system of D4 branes attached to it. By passing through the $\mathrm{NS}^{\prime}$ the O6 changes sign. In addition 4 half D6 branes and their mirrors start out from here and stretch to infinity. They also have a total D6 charge of +4 . It therfore seems to be reasonable to assume, that this whole system has a smooth M-theory interpretation. However this interpretation is still to be found. The half D6 branes are stuck at the orientifold.

As above, this system can be most easily analyzed by considering it as a projection of the product gauge group setup. Again the orientifold identifies the two gauge groups and the two adjoints. The degrees of freedom that lead to the bifundamentals turn into one antisymmetric and one conjugate symmetric tensor, since this time we have a different projection acting on the different sides of the D4 branes. In addition we have matter coming from the half D6 branes. Since they are stuck at the orientifold and the orientifold is stuck on top of the center $\mathrm{NS}^{\prime}$ brane, we necessarily have the half D6 branes on top of the $\mathrm{NS}^{\prime}$ brane and hence the issue of flavor doubling arises. This way we get an 
$S U\left(N_{c}\right)$ gauge group with one antisymmetric tensor $A$, one conjugate symmetric tensor $\tilde{S}$ and 8 fundamentals $T$. This gives rise to an anomaly free chiral theory! In addition there are $N_{f}$ vectorlike fundamental flavors $Q, \tilde{Q}$ arising from the D6 branes.

For simplicity we will only consider the $(1,1,1)$ case, that is only one NS brane of each type. The superpotential is

$$
W=\tilde{S} T T+\lambda X Q \tilde{Q}+m X^{2}+X A \tilde{S}
$$

where the first term is the superpotential required by the doubling mechanism, equation (1) and the rest is the remainig superpotential from the product gauge group (7) after projection. $m$ and $\lambda$ are again given in terms of the angles as above. For nonzero $m$ we can integrate out the adjoint and obtain quartic superpotential terms instead.

If we choose to move the additional D6 branes on top of the orientifold we again have to deal with doubling. From the brane configuration it is obvious that this way the global symmetry is enhanced to $S O\left(2 N_{f}+8\right) \times S p\left(N_{f}\right)$ if we are dealing with D6 branes and $S O(8) \times S O\left(2 N_{f}\right) \times S p\left(N_{f}\right)$ if we are dealing with $\mathrm{D} 6^{\prime}$ branes. For more general orientations of the D6 branes we are left with an $S O(8) \times S U\left(N_{f}\right) \times S U\left(N_{f}\right)$ global symmetry. To achieve this enhanced global symmetry in the field theory we add superpotential terms corresponding to (1) with the coefficients depending on the various rotation angles.

\subsubsection{Flat Directions}

The field theory considerations can again be found in $[19,18]$. Since we focus on the $(1,1,1)$ case we are dealing with a superpotential

$$
W=\tilde{S} T T+\lambda X Q \tilde{Q}+X A \tilde{S}+m X^{2}
$$

with $m=\tan \theta$ and $\lambda=\sin \omega$ with the angles defined as in figure 5 . Consider the case $\lambda=0$. For non-zero $m$ we can integrate out the massive adjoint and obtain

$$
W=\tilde{S} T T-\frac{1}{2 m}(A \tilde{S})^{2}
$$

For $\theta=0, \pi / 2$ the theory has a Coulomb branch. This is obvious from the brane picture. For $\theta=0$ all branes are parallel. The D4 branes are free to move in the 8,9 direction. In the field theory for $\theta=0$ the adjoint remains massless. It's vev parametrizes the Coulomb branch.

For $\theta=\pi / 2$ the $\mathrm{A}$ and $\mathrm{C}$ brane are antiparallel. This times the $\mathrm{D} 4$ branes are free to move in the 4,5 direction along the A and C NS branes. In the field theory this new 
branch opens up since the $(A \widetilde{S})^{2}$ term vanishes and now $A$ and $\tilde{S}$ are free to get vevs of the form

$$
\langle A\rangle=\sigma_{2} \otimes\left(\begin{array}{cccc}
x_{1} & & & \\
& x_{2} & & \\
& & \ddots & \\
& & & x_{n}
\end{array}\right) .
$$

and

$$
\langle\tilde{S}\rangle=\mathbf{1} \otimes\left(\begin{array}{cccc}
\tilde{x_{1}} & & & \\
& \tilde{x_{2}} & & \\
& & \ddots & \\
& & & \tilde{x}_{n}
\end{array}\right) .
$$

with $\left|x_{i}\right|^{2}-\left|\tilde{x}_{i}\right|^{2}=r$, where as above $r$ is associated with the position of the $\mathrm{NS}^{\prime}$ brane, generically breaking $S U\left(N_{c}\right)$ to $U(1)^{\frac{N_{c}}{2}}$ [19]. If $N_{c}$ is odd there is a zero in the lower right corner of $A$ and $\tilde{S}$. Note that on this Coulomb branch as opposed to the one associated with the adjoint, the rank of the gauge group is only half of the original rank. In the brane picture this is obvious, since in the 4,5 plane the orientifold is a point and hence the projection identifies a single brane and its mirror. If $N_{c}$ is odd one of the D4 branes is stuck at the orientifold.

For general values of $\theta$, the mass term for $X$ and the resulting quartic superpotential don't allow for any Coulomb branch. However there are several Higgs branches. The mesonic branches arise in the standard fashion by splitting the D4 branes between the D6 branes. The gauge group is broken to the same model with a lower rank gauge group.

More interesting are the baryonic branches of the theory. In the brane picture baryonic branches are associated with motion of NS branes in the 7 direction. Some of these baryonic branches already exist for $N_{f}=0$ and describe the possible deformations of the "fork" (the sign changing orientifold with the embedded $\mathrm{NS}^{\prime}$ brane and the 8 half D6 branes, figure 10$)$. Consider the flat direction along which the baryon $A^{n} \tilde{Q}^{N_{c}-2 n}$ gets an expectation value. The field theory analysis was performed in [19]. Along this flat direction the gauge group is broken to $S p(n)$ with a symmetric (adjoint) tensor and a superpotential $W=m X^{2}$. What happened in the brane language is that we moved the center NS' brane along the 7 direction. Thereby we also moved the point at which the orientifold changes sign. The NS - D4 system describing our remaining field theory now only sees the negatively charged orientifold with the embedded D6 branes, leading to an $N=2 S p(n)$ gauge theory for the case that the A and C branes are both NS. Rotating the A and C NS branes leads to the usual breaking of $N=2$ to $N=1$ by introducing the mass for the adjoint scalar in the $N=2$ vector multiplet. 
Similarly we can move the $\mathrm{NS}^{\prime}$ brane in the other direction, leaving us with an $S O(n)$ $\left(n<N_{c}\right)$ gauge theory with an adjoint and a superpotential $W=m X^{2}$ for this adjoint. In the field theory this is also a baryonic flat direction, this time associated with the baryon operator $\tilde{S}^{n} Q^{N_{c}-n} Q^{N_{c}-n}$. The $8 T$ fields no longer contribute any massless matter to the brane configuration, since our gauge system now is at a finite distance from the half D6 branes. In the field theory this effect is realized by the STT superpotential, giving the $T$ fields a mass proportional to the vev of $S$.

\subsubsection{Non-Abelian duality}

Since in the discussion of non-Abelian duality only the charge of the orientifold mattered, the brane picture seems to suggest that this chiral theory is again described by a dual $S U\left(3 N_{f}+4-N_{c}\right)$ gauge group with the same charged matter content and the obligatory mesons, since the complicated object stuck on top of the center $\mathrm{NS}^{\prime}$ brane has charge +4 on both sides, even though this charge arises in a different fashion on both sides. This kind of theory without the $\tilde{S} T T$ term was also analyzed in [18]. There, the dual is found to be an $S U\left(3\left(N_{f}+4\right)-N_{c}\right)$ gauge theory. To compare with our field theory prediction we have to analyze the effect of the $\tilde{S} T T$ perturbation in field theory. In the dual theory this operator is mapped to a meson $M$ that couples to the charged magentic fields via a superpotential [18]

$$
W=\ldots+M t \tilde{s} t+\ldots
$$

where the small letter fields transform under the same representations under the dual gauge group as their capital counterparts under the original gauge group. Perturbing this superpotential with the operator $\tilde{S} T T$ means that we add $M$ to the dual superpotential. Via the equations of motion for $M$ this forces that $t \tilde{s} t$ has non- vanishing vev. This kind of vev was analyzed by [21]. It was found that the dual group is broken by 1 unit per $t$ flavor involved, that is in our case by 8 units. The remaining dual gauge group is $S U\left(3 N_{f}+4-N_{c}\right)$ in perfect agreement with the brane prediction.

\subsection{Generalizations to Mutiple NS branes}

Similar to the product gauge group case we can generalize our setup to $(k, k, k)$ or $(k, 1, k)$. We would expect that this corresponds to chiral theories with a superpotential (for $\omega=0$ )

$$
W=m X^{k+1}+X A \tilde{S}+\tilde{S} T T
$$


and

$$
W=\frac{1}{2 m}(A \tilde{S})^{k+1}+\tilde{S} T T
$$

and similarly for the non-chiral theories discussed in section 4.3. These theories correspond to the $D_{k+2}$ and $A_{k}$ models in the classification of gauge theories with 2-index tensors of [18]. The D-type model with $k=2$ and $N_{f}=N_{c}$ gives rise to a chiral finite theory and was analyzed in [22]. A better understanding may shed some light on how finiteness is visible in the $N=1$ brane setup.

Even though this approach seems very promising, there are some discrepencies that need further understanding. To highlight this it is enough to consider the easier case of $(k, 0, k)$ as they arise on the baryonic branches of our models. That is, for an $\mathrm{O}^{\prime}$ we consider a $S O\left(N_{c}\right)$ gauge group with an antisymmetric tensor, $N_{f}$ flavors and superpotential $W=$ $A^{k+1}$, while for the $\mathrm{O} 6$ we consider a $S O\left(N_{c}\right)$ gauge group with a symmetric tensor, $N_{f}$ flavors and superpotential $W=S^{k+1}$. These gauge theories arise on the baryonic branch of the chiral and non-chiral models considered in section 4 respectively. In the language of [18] they correspond to the $A_{k}$ mezzanine and orchestra models respectively. Similarly one could discuss the corresponding $S p$ groups.

In the field theory analysis of $[19,18]$ it is found that one should restrict oneself to $k$ odd in the case of $S O$ with the antisymetric tensor in order to truncate the chiral ring and get a handle on the gauge theory. On the other hand for $S O$ with the symmetric no such restriction exists. It is not clear how this translates into the brane picture. This is a field theory prediction for the brane configuration which deserves further study.

If one tries to describe non-Abelian duality the branes give us the prediction that the dual gauge group should be $k N_{f}-N_{c}+$ const. where the constant is associated to the orientifold. The field theory tells us that the duals actually have $k N_{f}-N_{c}+4$ and $k N_{f}-N_{c}+4 k$ as their dual gauge theory. ${ }^{2}$. Note that while we get the factor in front of $N_{f}$ right, the orientation of the $\mathrm{O} 6$ affects the multiplicity of the +4 in the dual gauge group.

The same comments apply to the other setups. The factors of $(2 k+1) N_{f}$ in the $A_{k}$ balcony (keeping the relabelling in mind) and and $S U$ mezzanine models of [18] as well as the $3 k N_{f}$ in the $D_{k+2}$ series can be easily obtained from just translating the results of the product gauge group analysyis of [4]. However the effect of the orientifold charge and of the restrictions on $k$ to be odd in some cases deserve a further investigation.

\footnotetext{
${ }^{2}$ Keep in mind that for the mezzanine model we relabelled $2(\mathrm{k}+1)$ from the field theory to $(\mathrm{k}+1)$, since this is the $\mathrm{k}$ that is associated with the number of 5 branes in the brane picture
} 


\section{Small Instanton Transition and Chiral non-Chiral Transition}

We now turn to a very interesting effect which arises in the brane configuration. This effect was demonstrated in the context of six dimensional gauge theories in [9] and was called a "small instanton transition". It demonstrates the effect in which a tensor multiplet is traded with 29 hypermultiplets for a class of six dimensional models. Following [23, 24] this effect was first found in [25] and further discussed in [26]. We repeat the same brane motion for our four dimensional system. The results are very striking. We get a transition between two models, a chiral model and a non-chiral model. Moreover, this result is generic, as any brane configuration for a chiral theory has such a transition. We expect very interesting connections to arise from this effect.
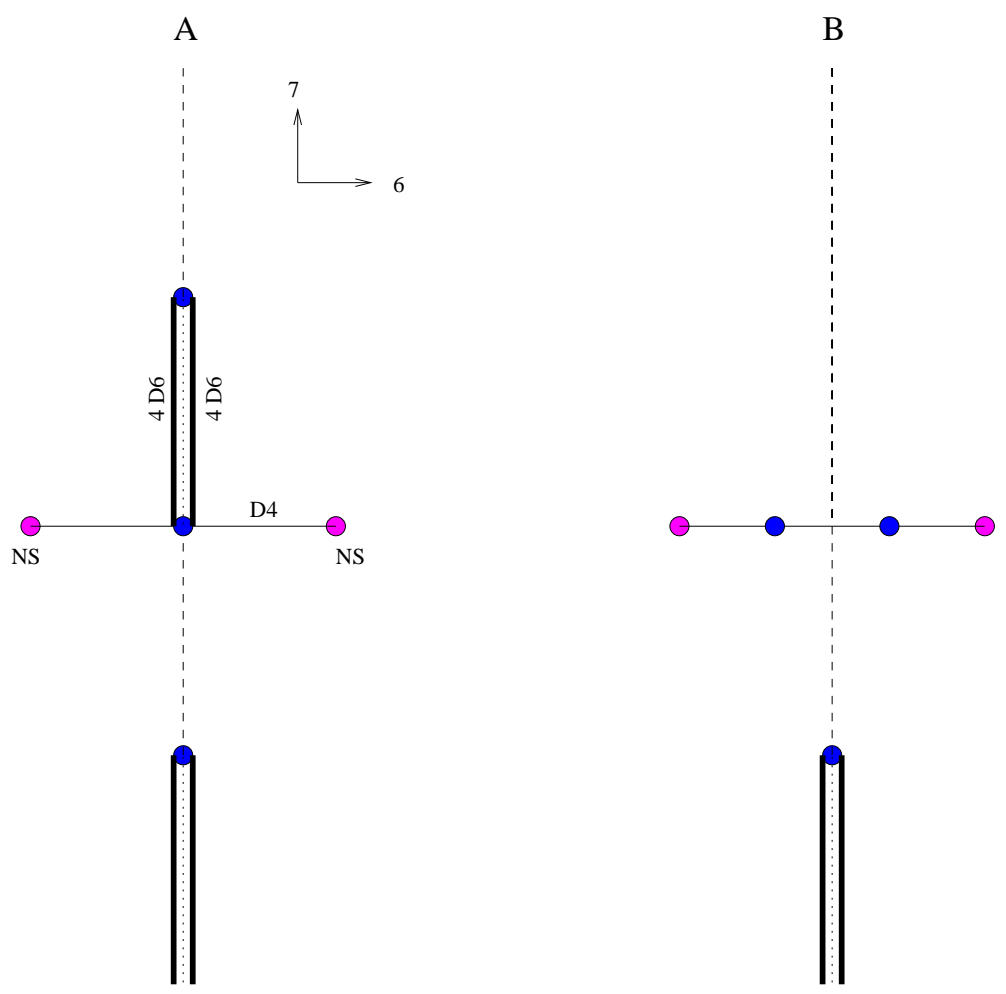

Figure 11: A small instanton transition which lead to chirality change in the spectrum. A NS' brane comes from infinity in the $x^{7}$ direction and combines with the stuck $\mathrm{NS}^{\prime}$ at the origin. At this point they can both leave the origin in the $x^{6}$ direction. The resulting four dimensional theory is no longer chiral. 
Consider the configuration depicted in figure $11 \mathrm{~A}$. It describes our chiral theory from section 4.4. Note, however that there are two additional $\mathrm{NS}^{\prime}$ branes in the system. We will call them upper and lower branes, respectively. The original NS' brane, to which the four dimensional system is attached, will be called central. When these two NS' branes are far away from the central NS' brane, they can not affect the dynamics of our theory and the model is just the model studied in section 4.4.

As we did for the case with no orientifold plane, it is useful to think of the underlying six dimensional theory which gives the global symmetries of the four dimensional system. This theory can be read off from the brane configuration and is just $S O(8)$. If, in addition, there are $N_{f}$ D6 branes on top of the orientifold plane, the gauge group is $S O\left(8+N_{f}\right) \times S p\left(N_{f}\right)$. The matter content of this theory has one bi-fundamental hypermultiplet, $N_{f}(\square, 1)$ and $N_{f}+8(1, \square)$. Each gauge group couples to a tensor multiplet which serves as a gauge coupling. The vev of the scalars in the two tensor multiplets determine the distance between the two pairs of adjacent $\mathrm{NS}^{\prime}$ branes.

The $\mathrm{NS}^{\prime}$ branes are not allowed to move in the 456 directions. This is because they carry one unit of charge and the orientifold allows for motion outside only in the case that an image exists. From a six dimensional point of view, this corresponds to the fact that there are no FI terms for $S O$ or $S p$ groups. On the other hand, the relative motion of the $\mathrm{NS}^{\prime}$ branes along the $x^{7}$ direction corresponds to a change in the two tensor multiplets in the thoery. There is, however, an option for a pair of $\mathrm{NS}^{\prime}$ branes to move in the 456 directions. We can move two $\mathrm{NS}^{\prime}$ branes to touch in the $x^{7}$ direction. At this point, the orientifold planes from above and below the pair of NS' branes are identical, as is clear from figure 11 . From a six dimensional point of view such a motion corresponds to taking one of the gauge couplings to infinity and thereby obtain strings with vanishing tension a non trivial fixed point. The pair of $\mathrm{NS}^{\prime}$ branes can then move in the 456 direction, as in figure $11 \mathrm{~B}$. The resulting six dimensional gauge group is now completely broken.

Let us go back to reinterpret this transition in our four dimensional system. When the NS' branes are far, we have our chiral theory. When the $\mathrm{NS}^{\prime}$ branes move in the 456 directions, the theory is different. We can read it off from figure $11 \mathrm{~B}$. We have $S O\left(N_{c}\right) \times S U\left(N_{c}\right)$ with a symmetric tensor for the $S O$ group and a pair (chiral and its conjugate) of bifundamental fields. The symmetric tensor arises as described in section 4.2. In addition we expect a superpotential $W=S^{2}+S \tilde{F} F$.

This is done when we take the upper $\mathrm{NS}^{\prime}$ brane and move out with the central $\mathrm{NS}^{\prime}$ brane. We can do it, instead, with the lower $\mathrm{NS}^{\prime}$ brane. The resulting gauge theory is $S p\left(\frac{N_{c}}{2}\right) \times S U\left(N_{c}\right)$ with an antisymmetric tensor for the $S p$ and a bi-fundamental pair. 
For this case, note that $N_{c}$ must be even, otherwise the motion of the $\mathrm{NS}^{\prime}$ branes to the 456 directions is not allowed and possibly breaks supersymmetry.

Here, we specify the matter content for the case when $N_{f}=0$. When $N_{f} \neq 0$, we need to take into account the effect of flavor doubling as discussed in section 3.1. It is straight forward and we will not repeat it here. There is however a difference for the $S p$ case. The upper 8 stuck D6 branes are combined with the lower 8 stuck D6 branes. At this point they are no longer stuck and give rise to 4 physical D6 branes, free to move in the 456 directions. The number of flavors for the $S p\left(\frac{N_{c}}{2}\right) \times S U\left(N_{c}\right)$ group is increased from $N_{f}$ to $N_{f}+4$.

To summarize, the analog of the six dimensional small instanton transition gives rise to a transition between a chiral theory with a $S U\left(N_{c}\right)$ gauge theory with an antisymmetric tensor, one conjugate symmetric tensor and 8 fundamentals to either

- a $S O\left(N_{c}\right) \times S U\left(N_{c}\right)$ gauge group with matter transforming as $(\square, 1),(\square, \square)$ and $(\square, \bar{\square})$

- or a $S p\left(\frac{N_{c}}{2}\right) \times S U\left(N_{c}\right)$ gauge group with matter transforming as $(\boxminus, 1),(\square, \square),(\square, \bar{\square})$ and $8(\square, 1)$.

In addition one can include an arbitrary number of flavors from D6 branes. At the point in the transition when all the $\mathrm{NS}^{\prime}$ branes meet we find in the field theory a non-trivial fixed point with enhanced global symmetry. In the first case the global symmetry at the fixed point solely comes from the coinciding $\mathrm{NS}^{\prime}$ branes. In the second case in addition to this the $S O(8)$ flavor rotations are enhanced to $S O(8) \times S O(8)$ at the fixed point.

Acknowledgements

We would like to thank John Brodie and Alberto Zaffaroni for useful discussions. A.H. would like to thank the Institut für Physik at the Humboldt Universität for their hospitality while this work was being completed. The research of A.H. is supported in part by NSF grant PHY-9513835 and the research of I.B. and A.K. is supported by Deutsche Forschungsgemeinschaft.

\section{References}

[1] A. Hanany and E. Witten, "Type IIB superstrings, BPS monopoles, and three-dimensional gauge dynamics," Nucl. Phys. B492 (1997) 152-190, hep-th/9611230. 
[2] N. Seiberg, "Electric - magnetic duality in supersymmetric nonabelian gauge theories," Nucl. Phys. B435 (1995) 129-146, hep-th/9411149.

[3] S. Elitzur, A. Giveon, and D. Kutasov, "Branes and N=1 duality in string theory," Phys. Lett. B400 (1997) 269-274, hep-th/9702014.

[4] J. H. Brodie and A. Hanany, "Type IIA superstrings, chiral symmetry, and N=1 4-d gauge theory dualities," Nucl. Phys. B506 (1997) 157, hep-th/9704043.

[5] A. Hanany and A. Zaffaroni, "Chiral symmetry from Type IIA branes," hep-th/9706047.

[6] K. Landsteiner, E. Lopez, and D. A. Lowe, "Duality of chiral N=1 supersymmetric gauge theories via branes," hep-th/9801002.

[7] J. Lykken, E. Poppitz, and S. P. Trivedi, "Chiral gauge theories from D-branes," hep-th/9708134.

[8] J. Lykken, E. Poppitz, and S. P. Trivedi, "M(ore) on chiral gauge theories from D-branes," hep-th/9712193.

[9] A. Hanany and A. Zaffaroni, "Branes and six-dimensional supersymmetric theories," hep-th/9712145.

[10] S. Kachru and E. Silverstein, "Chirality changing phase transitions in 4-d string vacua," Nucl. Phys. B504 (1997) 272, hep-th/9704185.

[11] M. Klein and J. Louis, "Singular yukawa and gauge couplings in $\mathrm{d}=4$ heterotic string vacua," hep-th/9707212.

[12] O. Aharony and A. Hanany, "Branes, superpotentials and superconformal fixed points," hep-th/9704170.

[13] A. Hanany and K. Hori, "Branes and N=2 theories in two-dimensions," hep-th/9707192.

[14] I. Brunner and A. Karch, "Branes and six-dimensional fixed points," Phys. Lett. B409 (1997) 109-116, hep-th/9705022.

[15] I. Brunner and A. Karch, "Branes at orbifolds versus Hanany Witten in sixdimensions," hep-th/9712143. 
[16] C. Ahn, K. Oh, and R. Tatar, "Branes, geometry and $n=1$ duality with product gauge groups of s0 and sp," hep-th/9707027.

[17] K. Landsteiner and E. Lopez, "New curves from branes," hep-th/9708118.

[18] J. H. Brodie and M. J. Strassler, "Patterns of duality in N=1 SUSY gauge theories, or: Seating preferences of theater going nonabelian dualities," hep-th/9611197.

[19] K. Intriligator, R. G. Leigh, and M. J. Strassler, "New examples of duality in chiral and nonchiral supersymmetric gauge theories," Nucl. Phys. B456 (1995) 567, hep-th/9506148.

[20] N. Evans, C. V. Johnson, and A. D. Shapere, "Orientifolds, branes, and duality of 4-d gauge theories," Nucl. Phys. B505 (1997) 251, hep-th/9703210.

[21] P. Pouliot and M. J. Strassler, "Duality and dynamical supersymmetry breaking in Spin(10) with a spinor," Phys. Lett. B375 (1996) 175-180, hep-th/9602031.

[22] A. Karch, D. Lust, and G. Zoupanos, "Dualities in all order finite $\mathrm{N}=1$ gauge theories," hep-th/9711157.

[23] E. Witten, "Small instantons in string theory," Nucl. Phys. B460 (1996) 541-559, hep-th/9511030.

[24] N. Seiberg and E. Witten, "Comments on string dynamics in six-dimensions," Nucl. Phys. B471 (1996) 121-134, hep-th/9603003.

[25] P. S. Aspinwall, "Pointlike instantons and the spin (32) / $z_{2}$ heterotic string," Nucl. Phys. B496 (1997) 149, hep-th/9612108.

[26] K. Intriligator, "RG fixed points in six-dimensions via branes at orbifold singularities," Nucl. Phys. B496 (1997) 177, hep-th/9702038. 